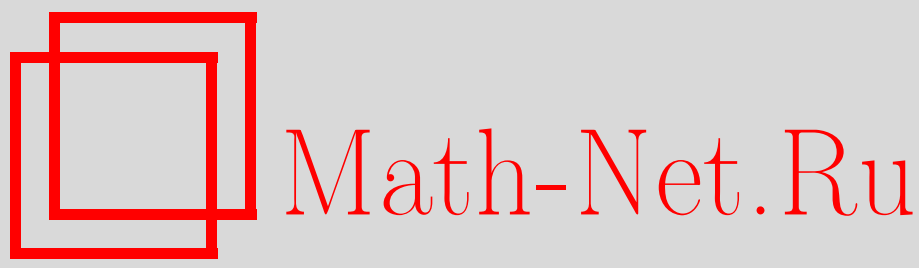

Д. Барриос, Г. Л. Лопес, А. Мартинес-Финкельштейн, Е. Торрано, Конечномерные аппроксимации резольвенты бесконечной ленточной матрицы и непрерывные дроби, Матем. сб., 1999, том 190, номер 4, 23-42

DOI: https://doi.org/10.4213/sm389

Использование Общероссийского математического портала Math-Net.Ru подразумевает, что вы прочитали и согласны с пользовательским соглашением

http://www . mathnet.ru/rus/agreement

Параметры загрузки:

IP: 52.6 .47 .48

26 апреля 2023 г., 17:31:17 
Д. Барриос, Г. Лопес, А. Мартинес-Финкельштейн, Э. Торрано

\title{
Конечномерные аппроксимации резольвенты бесконечной ленточной матрицы и непрерывные дроби
}

\begin{abstract}
В работе исследуется вопрос о возможности аппроксимации резольвенты оператора, порожденного ленточной матрицей, посредством резольвент конечномерных срезов этой матрицы. Дается положительный ответ для матриц, допускающих представление в виде суммы самосопряженной и ограниченной ленточных матриц. В этом случае область сходимости последовательности резольвент описьвается в терминах матриц, входящих в представление. В применении к комплексным трехдиагональным матрицам этот результат позволяет установить достаточные условия сходимости чебьшевских непрерывных дробей на множествах комплексной области. В частном случае компактных возмущений результат уточняется и устанавливается связь между полюсами предельной функции и собственньми значениями трехдиагональной матрицы.
\end{abstract}

Библиограф̆ия: 13 названий.

\section{$\S 1$. Введение и формулировка основного результата}

Хорошо известно, что свойства непрерьвной дроби можно исследовать, изучая некоторый оператор, порожденньй трех диагональной (якобиевой) матрицей, построенной по коэффициентам непрерывной дроби. Этот метод широко применялся до сих пор, и краткость доказательства известных результатов является сильньгм аргументом в пользу такого подхода.

В случае действительной симметричной трех диагональной матрицы основными инструментами исследования являются классическая проблема моментов, теория ортогональных многочленов и спектральная теория самосопряженных операторов (см., например, [1], [2]).

Если же обратиться к непрерывным дробям с комплексньми коэффициентами или же к аппроксимациям Эрмита-Паде (помимо других примеров), то возникают неэрмитовы либо нетрехдиагональные ленточные матрицы. В этом случае, как правило, невозможно использовать спектральную теорему или ортогональные многочлены и приходится применять иную технику.

В настоящей работе, состоящей из четырех параграфов, мы стремимся получить определенное обобщение классической теории, рассматривая ограниченное возмущение неограниченного самосопряженного оператора в случае, ког да оба оператора порождаются ленточными матрицами (см. $\S \S 1$ и 2). Мы применяем полученные результаты к классу чебышевских непрерьвных дробей с целью получить условия сходимости на множествах комплексной плоскости. В $\S 4$, где рассматривается 
частный случай компактных возмушений, этот результат уточняется и устанавливается взаимосвязь между полюсами предельной функции и собственными значениями трех диагональной матрицы.

Здесь мы в основном интересуемся непрерывными дробями. Некоторые дополнительные применения основного результата (теоремы 1 ) будут опубликованы позже.

В оставшейся части этого параграфа мы вводим необходимые определения и формулируем результаты о сходимости операторов.

Рассмотрим бесконечную ленточную или $(2 p+1)$-диагональную матрицу

$$
G=\left(g_{i j}\right)_{i, j=0}^{\infty}
$$

где $g_{i j}=0$ при $|i-j|>p$. Для любой последовательности комплексных чисел $x=\left(x_{1}, x_{2}, \ldots\right)$ обозначим через $G x$ последовательность с компонентами $(G x)_{n}$, получаемую формальным умножением $G$ на вектор $x$.

Чтобы перейти от матрицы $G$ к операторам, введем следующие линейные подмножества гильбертова пространства $\ell^{2}: D_{0}$, состоящее из векторов с конечным числом ненулевых компонент, и $D(G)=\left\{x \in \ell^{2}: G x \in \ell^{2}\right\}$; оба множества плотны в $\ell^{2}, D_{0} \subset D(G)$ в силу ленточной структуры $G$ (см. [3]). Определим оператор $\mathscr{G}$ на $D(G)$ равенством $\mathscr{G}(x)=G x$ и обозначим через $\mathscr{G}_{0}$ ограничение $\mathscr{G}$ на $D_{0}$. Операторы $\mathscr{G}_{0}$ и $\mathscr{G}$ будем называть соответственно минимальным и максимальным операторами, порожденньми матрицей $G$ (несколько иное определение см., например, в [4]). В дальнейшем, там, где это не приведет к недоразумениям, мы будем использовать для матрицы и для максимального оператора $\mathscr{G}$ единое обозначение $G$.

Ленточная матрица $G$ порождает ограниченный оператор в $\ell^{2}$ тогда и только тогда, когда все ее элементы $g_{i j}$ равномерно ограничены; в этом случае указанное матричное представление оператора имеет смысл для всех элементов $x \in \ell^{2}$, и такие матрицы мы называем ограниченными. В частности, если ограниченная матрица $G$ эрмитова (т.е. $g_{i j}=\overline{g_{j i}}$ ), то $\mathscr{G}$ является самосопряженным оператором.

В обшем (неограниченном) случае для эрмитовой матрицы $G$ по крайней мере можно утверждать, что $\mathscr{G}_{0}$ симметричен и имеет замькание, которое обозначим через $\mathscr{G}_{0}^{\prime}$. Нетрудно установить, что оператор $\mathscr{G}$ сопряжен к $\mathscr{G}_{0}^{\prime}: \mathscr{G}=\left[\mathscr{G}_{0}^{\prime}\right]^{*}$. Если $\mathscr{G}$ самосопряжен (другими словами, если $\mathscr{G}=\mathscr{G}_{0}^{\prime}$ ), мы говорим, что матрица $G$ является самосопряженной.

Пусть $\left\{e_{i}\right\}_{i=0}^{\infty}, e_{i}=(0,0, \ldots, 0, \overbrace{1}^{(i+1)}, 0, \ldots)^{T}$, обозначает стандартный базис в $\ell^{2}$. Для каждого фиксированного $n \in \mathbb{N}$ рассмотрим оператор $E_{n}: \ell^{2} \rightarrow \mathbb{C}^{n}$, определяемый равенством $E_{n} x=\left(x_{1}, x_{2}, \ldots, x_{n}\right)$ для каждого $x=\left(x_{1}, x_{2}, \ldots\right) \in$ $\ell^{2}$, и оператор $\widetilde{E}_{n}: \mathbb{C}^{n} \rightarrow \ell^{2}$, для которого

$$
\widetilde{E}_{n}\left(x_{1}, x_{2}, \ldots, x_{n}\right)=\left(x_{1}, x_{2}, \ldots, x_{n}, 0, \ldots\right) \in \ell^{2} .
$$

Очевидно, матричное представление оператора $\widetilde{E}_{n} E_{n}: \ell^{2} \rightarrow \ell^{2}$ в базисе $\left\{e_{i}\right\}$ имеет вид блочной матрицы

$$
\widetilde{E}_{n} E_{n}=\left(\begin{array}{cc}
I_{n} & 0 \\
0 & 0
\end{array}\right)_{\infty \times \infty} \quad, \quad \text { где } I_{n}=\left(\begin{array}{ccc}
1 & & \\
& \ddots & \\
& & 1
\end{array}\right)_{n \times n}
$$


причем $E_{n} \widetilde{E}_{n}=I_{n}: \mathbb{C}^{n} \rightarrow \mathbb{C}^{n}$.

Для бесконечной матрицы $G=\left(g_{i j}\right)_{i, j=0}^{\infty}$ определим конечномерные "срезы" $G_{n}=\left(g_{i j}\right)_{i, j=0}^{n-1}$; другими словами, $G_{n}$ - это матрица размера $n \times n$, составленная из первых $n$ строк и $n$ столбцов $G$. Каждая матрица $\widetilde{E}_{n} G_{n} E_{n}$ порождает ограниченный оператор конечного ранга в $\ell^{2}$, матрица которого имеет вид

$$
\left(\begin{array}{cc}
G_{n} & 0 \\
0 & 0
\end{array}\right)_{\infty \times \infty}
$$

В дальнейшем мы отождествляем спектр ленточной матрицы $G$ со спектром порожденного ею максимального оператора. Резольвентное множество $\rho(G)$ матрицы $G$ состоит из $z \in \mathbb{C}$, для которых ядро оператора $G-z I$ тривиально, его образ совпадает с $\ell^{2}$ и $(G-z I)^{-1}$ ограничен на $\ell^{2}$. Далее, $\sigma(G)=\mathbb{C} \backslash \rho(G)$ обозначает спектр, а $\sigma_{p}(G) \subset \sigma(G)$ - точечный спектр (совокупность собственных значений) $G$.

Таким образом, резольвентный оператор

$$
\mathscr{R}^{(G)}(z)=(G-z I)^{-1}
$$

определен и ограничен на $\ell^{2}$ для $z \in \rho(G)$. Кроме того, если $z \notin \sigma\left(G_{n}\right)$, то $\left(G_{n}-z I_{n}\right)^{-1}$ биективен на $\mathbb{C}^{n}$, а оператор

$$
\mathscr{R}_{n}^{(G)}(z)=\widetilde{E}_{n}\left(G_{n}-z I_{n}\right)^{-1} E_{n}
$$

ограничен на $\ell^{2}$.

Положим

$$
\mathscr{P}(G)=\bigcap_{n \geqslant 0} \overline{\bigcup_{k \geqslant n} \sigma\left(G_{k}\right)} .
$$

Очевидно, $\mathscr{P}(G)$ можно охарактеризовать как множество всех $z \in \mathbb{C}$, для которых существует такая последовательность $\left\{z_{n}\right\}_{n \in \Lambda}, z_{n} \in \sigma\left(G_{n}\right), n \in \Lambda \subset \mathbb{N}$, что

$$
\lim _{n \in \Lambda} z_{n}=z
$$

В (классическом) случае действительной якобиевой матрицы $G$, как известно, $\sigma(G) \subset \mathscr{P}(G) \subset \operatorname{conv} \sigma(G)$ (см., например, [5; гл. II, раздел 4]).

Наконец, под $\operatorname{dist}(z, A)$ мы понимаем евклидово расстояние в $\mathbb{C}$ от точки $z$ до множества $A,\|\cdot\|$ обозначает обычную норму в $\ell^{2},\|\cdot\|_{n}$ обозначает евклидову норму в $\mathbb{C}^{n}$, а знак “ $\longrightarrow$ ” в применении к операторам указывает на сильную (или обычную) сходимость.

Все наши последующие рассмотрения относятся к случаю, когда матрица $G$ может бить представлена в виде

$$
G=H+C
$$

где ленточные матриць $H$ и $С$ являются соответственно самосопряженной и ограниченной. Следует обратить внимание на определенную свободу в выборе матриц $H$ и $C$. 
TEOPEMA 1. Eсли имеет место (2), mo

$$
\mathscr{R}_{n}^{(G)}(z) \rightarrow \mathscr{R}^{(G)}(z)
$$

равномерно на компактах в $\{z: \operatorname{dist}(z, \mathscr{P}(H) \cup \sigma(H))>\|C\|\}$.

В частности, так как все $\sigma\left(H_{n}\right) \subset \mathbb{R}, \sigma(H) \subset \mathbb{R}$, можно прийти к следуюшему заключению.

СлЕДСТВИЕ 1. Если имеет место (2), то справедливо (3) равномерно на компактах в $\{z:|\operatorname{Im} z|>\|C\|\}$.

В дополнение для трех диагональных матриц можно доказать более точный результат. В этом случае известно, что все $\sigma\left(H_{n}\right)$ принадлежат выпуклой оболочке conv $\sigma(H)$ множества $\sigma(H)$, откуда получаем

СлЕДСтВИЕ 2. Если матрица $Н$ трехдиагональна, то при условии (2) имеет место (3) равномерно на любом компакте $K$, удовлетворяющем неравенствв $y \operatorname{dist}(K, \operatorname{conv} \sigma(H))>\|C\|$.

Обозначим через $S$ правый сдвиг на $\ell^{2}$, порождаемый бесконечной матрицей

$$
S=\left(\begin{array}{cccc}
0 & 0 & 0 & \ldots \\
1 & 0 & 0 & \ldots \\
0 & 1 & 0 & \ldots \\
\vdots & \vdots & \vdots & \ddots
\end{array}\right)_{\infty \times \infty}
$$

и пусть $G^{(k)}=\left[S^{k}\right]^{T} G S^{k}, k=1,2, \ldots$. Таким образом, матрица $G^{(k)}$ получается отбрасыванием у $G$ первых $k$ строк и $k$ столбцов. Так как при каждом $k$ матрица $G^{(k)}$ сохраняет структуру, указанную в $(2), G^{(k)}=H^{(k)}+C^{(k)}$, получаем

СлЕДСТВИЕ 3. При условии (2) для каждого $k=1,2, \ldots$

$$
\mathscr{R}_{n}^{\left(G^{(k)}\right)}(z) \rightarrow \mathscr{R}^{\left(G^{(k)}\right)}(z) \quad n p u \quad n \rightarrow \infty
$$

равномерно на компактах в $\left\{z: \operatorname{dist}\left(z, \mathscr{P}\left(H^{(k)}\right) \cup \sigma\left(H^{(k)}\right)\right)>\left\|C^{(k)}\right\|\right\}$.

В случае $H=0$ имеем

СЛЕДСТВИЕ 4. Если $\|G\|<\infty$, то (3) имеет место равномерно на комnактах в $\{|z|>\|G\|\}$.

\section{§ 2. Доказательство теоремы 1}

Мы разделим доказательство основного результата на несколько лемм, представляющих самостоятельный интерес.

Лемма 1. Если $\|C\|<\infty$, mо $\widetilde{E}_{n} C_{n} E_{n} \rightarrow C$, әде $C_{n}=E_{n} C \widetilde{E}_{n}$. 
ДокаЗАтЕльство. Так как оператор $\widetilde{E}_{n} E_{n}$ является проектором из $\ell^{2}$ на $\operatorname{span}\left\{e_{0}, \ldots, e_{n-1}\right\}$, a $C_{n}=E_{n} C \widetilde{E}_{n}$, то $\widetilde{E}_{n} C_{n} E_{n}=\widetilde{E}_{n} E_{n} C \widetilde{E}_{n} E_{n}$. Таким образом, $\left\|\widetilde{E}_{n} C_{n} E_{n}\right\| \leqslant\|C\|$. С другой стороны,

$$
\left(C-\widetilde{E}_{n} C_{n} E_{n}\right) e_{j}=\left(I-\widetilde{E}_{n} E_{n}\right) C e_{j} \rightarrow 0, \quad n \rightarrow \infty,
$$

где $C e_{j}-j$-й столбец матрицы $C$. Следовательно, величины $\left\|\widetilde{E}_{n} C_{n} E_{n}\right\|$ равномерно ограничены и имеет место равномерная сходимость на плотном подмножестве в $\ell^{2}$.

Лемма 2. Ecли $\operatorname{dist}(z, \sigma(H))>\|C\|$, то $z \in \rho(G)$. Кроме того,

$$
\left\|\mathscr{R}^{(G)}(z)\right\| \leqslant \frac{1}{\operatorname{dist}(z, \sigma(H))-\|C\|} .
$$

ДокАЗАТЕЛьСтво. При условии $\|G\|<\infty$ (или, эквивалентно, $\|H\|<\infty$ ) утверждение является элементарньм следствием известной теоремы об обратимости малых возмущений ограниченного обратимого оператора. В общем случае мы используем тождество

$$
\mathscr{R}^{(G)}(z)=\mathscr{R}^{(H)}(z)\left[I+C \mathscr{R}^{(H)}(z)\right]^{-1}
$$

справедливое везде, где определены входящие в него операторы, а также тот факт, что для самосопряженного оператора $H$ и $z \in \rho(H)$

$$
\left\|\mathscr{R}^{(H)}(z)\right\|=\frac{1}{\operatorname{dist}(z, \sigma(H))}
$$

(см., например, [6; задача III-6.16]). Таким образом, если $\operatorname{dist}(z, \sigma(H))>\|C\|$, то

$$
\left\|C \mathscr{R}^{(H)}(z)\right\| \leqslant\|C\| \frac{1}{\operatorname{dist}(z, \sigma(H))}<1,
$$

а значит, оператор $\left[I+C \mathscr{R}^{(H)}(z)\right]^{-1}$ сушествует и ограничен, т.е. $\mathscr{R}^{(G)}(z)$ является композицией ограниченных операторов.

Далее, из тождества

$$
\mathscr{R}^{(H)}(z)-\mathscr{R}^{(G)}(z)=\mathscr{R}^{(H)}(z) C \mathscr{R}^{(G)}(z)
$$

следует, что если $z \in \rho(H) \cap \rho(G)$, то

$$
\left\|\mathscr{R}^{(G)}(z)\right\| \leqslant\left\|\mathscr{R}^{(H)}(z)\right\|+\left\|\mathscr{R}^{(H)}(z)\right\| \cdot\|C\| \cdot\left\|\mathscr{R}^{(G)}(z)\right\|,
$$

откуда

$$
\left\|\mathscr{R}^{(G)}(z)\right\|\left(1-\|C\| \cdot\left\|\mathscr{R}^{(H)}(z)\right\|\right) \leqslant\left\|\mathscr{R}^{(H)}(z)\right\|,
$$

и остается применить (7).

Следующий шаг состоит в доказательстве равномерной ограниченности норм $\mathscr{R}_{n}^{(G)}(z)$, а именно имеет место 
ЛЕмма 3. Для достаточно больиих п величинь $\left\|\mathscr{R}_{n}^{(G)}(z)\right\|$ равномерно ограничены на компактах из $\{z: \operatorname{dist}(z, \mathscr{P}(H))>\|C\|\}$.

ДокАЗАТЕЛЬСТво. Поскольку $\left\|\mathscr{R}_{n}^{(G)}(z)\right\| \leqslant\left\|\left(G_{n}-z I_{n}\right)^{-1}\right\|_{n}$, то, следуя схеме доказательства леммы 2 , заключаем, что при $\operatorname{dist}\left(z, \sigma\left(H_{n}\right)\right)>\left\|C_{n}\right\|_{n}$

$$
\left\|\mathscr{R}_{n}^{(G)}(z)\right\| \leqslant \frac{1}{\operatorname{dist}\left(z, \sigma\left(H_{n}\right)\right)-\left\|C_{n}\right\|_{n}} .
$$

В силу леммы $1\left\|C_{n}\right\|_{n}=\left\|\widetilde{E}_{n} C_{n} E_{n}\right\| \rightarrow\|C\|$. С другой стороны, для любого $\varepsilon>0$ и всех достаточно больших $n$

$$
\operatorname{dist}\left(z, \sigma\left(H_{n}\right)\right) \geqslant \operatorname{dist}(z, \mathscr{P}(H))-\varepsilon
$$

Следовательно, можно утверждать, что

$$
\left\|\mathscr{R}_{n}^{(G)}(z)\right\| \leqslant \frac{2}{\operatorname{dist}(z, \mathscr{P}(H))-\|C\|}, \quad n \geqslant n_{0}(z)
$$

Кроме того, в силу резольвентного уравнения

$$
\mathscr{R}^{(G)}(z)-\mathscr{R}^{(G)}\left(z_{0}\right)=\left(z-z_{0}\right) \mathscr{R}^{(G)}(z) \mathscr{R}^{(G)}\left(z_{0}\right), \quad z, z_{0} \in \rho(G),
$$

имеет место неравенство

$$
\left\|\mathscr{R}^{(G)}(z)\right\| \leqslant \frac{\left\|\mathscr{R}^{(G)}\left(z_{0}\right)\right\|}{1-\left|z-z_{0}\right|\left\|\mathscr{R}^{(G)}\left(z_{0}\right)\right\|}, \quad\left|z-z_{0}\right|<\frac{1}{\left\|\mathscr{R}^{(G)}\left(z_{0}\right)\right\|} .
$$

Отсюда получаем, что $\mathscr{R}^{(G)}(z)$ равномерно ограничен на дисках

$$
\left\{z:\left|z-z_{0}\right| \leqslant \delta\left(z_{0}\right)\right\}, \quad \delta\left(z_{0}\right)<\frac{1}{\left\|\mathscr{R}^{(G)}\left(z_{0}\right)\right\|}
$$

для любого $z_{0} \in\{z: \operatorname{dist}(z, \mathscr{P}(H))>\|C\|\}$, и стандартные аргументы позволяют установить равномерную ограниченность на указанных вьше компактах.

Установим теперь сходимость в самосопряженном случае. Как будет показано ниже, следуюшая лемма является обобшением классической теоремы Стилтьеса о сходимости непрерывных дробей.

Лемма 4. Равномерно на компактных подмножествах в $\rho(H) \backslash \mathscr{P}(H)$

$$
\mathscr{R}_{n}^{(H)}(z) \rightarrow \mathscr{R}^{(H)}(z)
$$


ДокАЗАТЕльство. Для $z \in \rho(H)$ определим

$$
v_{j}=(H-z I) e_{j}, \quad j=0,1, \ldots
$$

Так как $z \in \rho(H)$, то

$$
\mathscr{R}^{(H)}(z) v_{j}=e_{j} .
$$

Для доказательства плотности $\operatorname{span}\left\{v_{j}, j=0,1, \ldots\right\}$ в $\ell^{2}$ достаточно установить, что

$$
\left\langle x, v_{j}\right\rangle=0, \quad x \in \ell^{2}, \quad j=0,1, \ldots,
$$

тогда и только тогда, когда $x=0$.

Для любого вектора $x$ справедливо следующее равенство

$$
\left\langle x,(H-z I) e_{j}\right\rangle=\left\langle(H-\bar{z} I) x, e_{j}\right\rangle, \quad j=0,1, \ldots .
$$

Следовательно, если имеет место (10), то

$$
\left\langle(H-\bar{z} I) x, e_{j}\right\rangle=0, \quad j=0,1, \ldots,
$$

откуда

$$
(H-\bar{z} I) x=0 .
$$

Заметим, что, вообще говоря, не любой вектор $(H-\bar{z} I) x$ обязан принадлежать $\ell^{2}$; однако если верно (11), то $H x=\bar{z} x$, откуда $H x \in \ell^{2}$, а следовательно (вспомним определение $D(H)), x \in D(H)$. Но $\mathscr{P}(H) \cup \sigma(H) \subset \mathbb{R}$, а значит, $\bar{z} \notin \sigma_{p}(H)$, в силу чего $x=0$.

С другой стороны, имеем

$$
E_{n} v_{j}=E_{n}(H-z I) e_{j}=\left(H_{n}-z I_{n}\right) E_{n} e_{j} \in \mathbb{C}^{n} \text { при } n \geqslant n_{0}(j), \quad j=0,1, \ldots
$$

Следовательно, для любого фиксированного $j=0,1, \ldots$

$$
\mathscr{R}_{n}^{(H)}(z) v_{j}=\widetilde{E}_{n}\left(H_{n}-z I_{n}\right)^{-1} E_{n} v_{j}=\widetilde{E}_{n} E_{n} e_{j}=e_{j}=\mathscr{R}^{(H)}(z) v_{j}
$$

при достаточно больших $n$ (точнее, для $n>j+p$ при условии, что матрица $H$ $(2 p+1)$-диагональна).

Таким образом, установлено, что

$$
\left[\mathscr{R}^{(H)}(z)-\mathscr{R}_{n}^{(H)}(z)\right] x \rightarrow 0, \quad n \rightarrow \infty,
$$

для $x$, принадлежащих плотному подмножеству $\ell^{2}$. Полагая $C=0$ в лемме 3 , получаем, что $\left\|\mathscr{R}_{n}^{(H)}(z)\right\|$ равномерно ограничены на каждом компакте в $\mathbb{C} \backslash \mathscr{P}(H)=$ $\rho(H) \backslash \mathscr{P}(H)$, откуда следует сходимость для $z \in \mathbb{C} \backslash \mathscr{P}(H)$. С учетом этого факта, неравенства

$$
\begin{aligned}
\left\|\mathscr{R}_{n}^{(H)}(z) x-\mathscr{R}^{(H)}(z) x\right\| \leqslant & \left\|\mathscr{R}_{n}^{(H)}(z) x-\mathscr{R}_{n}^{(H)}\left(z_{0}\right) x\right\|+\left\|\mathscr{R}_{n}^{(H)}\left(z_{0}\right) x-\mathscr{R}^{(H)}\left(z_{0}\right) x\right\| \\
& +\left\|\mathscr{R}^{(H)}\left(z_{0}\right) x-\mathscr{R}^{(H)}(z) x\right\|
\end{aligned}
$$

и резольвентного уравнения (9) (для $H$ и $H_{n}$ ) непосредственно получаем утверждение леммы.

Нам понадобится еще один технический результат: с целью установить сходимость “приближенных" обратных некоторых матриц докажем следующий вариант теоремы Канторовича (см., например, [7; гл. 14]). 
Лемма 5. Пусть $A$ - ограниченный обратимый оператор на $\ell^{2} ;$ рассмотрим последовательность обратимых операторов $A_{n}: \mathbb{C}^{n} \rightarrow \mathbb{C}^{n}$, удовлетворяющих условиям

$$
\widetilde{E}_{n} A_{n} E_{n} \rightarrow A
$$

$u$

$$
\left\|A_{n}^{-1}\right\|_{n} \leqslant K
$$

(константа $K$ не зависит от $n$ ). Тогда

$$
\widetilde{E}_{n} A_{n}^{-1} E_{n} \rightarrow A^{-1}
$$

ДоКАЗАТЕЛЬство. Последуем схеме, предложенной в работе [8; теорема 4.1]. Для любого $y \in \ell^{2}$ положим $x=A^{-1} y$. Так как $E_{n} \widetilde{E}_{n}=I_{n}: \mathbb{C}^{n} \rightarrow \mathbb{C}^{n}$, то

$$
\begin{aligned}
\widetilde{E}_{n} A_{n}^{-1} E_{n} y-A^{-1} y & =\widetilde{E}_{n} A_{n}^{-1} E_{n}\left(A x-\widetilde{E}_{n} A_{n} E_{n} x\right)+\left(\widetilde{E}_{n} A_{n}^{-1} E_{n} \widetilde{E}_{n} A_{n} E_{n} x-x\right) \\
& =\widetilde{E}_{n} A_{n}^{-1} E_{n}\left(A x-\widetilde{E}_{n} A_{n} E_{n} x\right)+\left(\widetilde{E}_{n} E_{n} x-x\right) .
\end{aligned}
$$

Очевидно, $\left(\widetilde{E}_{n} E_{n}-I\right) x \rightarrow 0$, а в силу (12) и (13) первое слагаемое также стремится к нулю.

Мы готовы теперь к доказательству основного результата этого параграфа.

Для $z$, удовлетворяющих условиям теоремы 1 , в силу леммы 4

$$
\mathscr{R}_{n}^{(H)}(z) \rightarrow \mathscr{R}^{(H)}(z)
$$

откуда, используя лемму 1 , получаем

$$
\widetilde{E}_{n} C_{n} E_{n} \mathscr{R}_{n}^{(H)}(z) \rightarrow C \mathscr{R}^{(H)}(z) .
$$

Таким образом, с учетом (7)

$$
\left\|C_{n}\left(H_{n}-z I_{n}\right)^{-1}\right\|_{n} \leqslant\left\|C_{n}\right\|_{n}\left\|\left(H_{n}-z I_{n}\right)^{-1}\right\|_{n} \rightarrow \frac{\|C\|}{\operatorname{dist}(z, \sigma(H))}<1,
$$

откуда

$$
\left\|C_{n}\left(H_{n}-z I_{n}\right)^{-1}\right\|_{n} \leqslant M<1, \quad n \geqslant n_{0}(z) .
$$

Следовательно, операторы $I_{n}+C_{n}\left(H_{n}-z I_{n}\right)^{-1}: \mathbb{C}^{n} \rightarrow \mathbb{C}^{n}$ имеют ограниченњые обратные и

$$
\left\|\left[I_{n}+C_{n}\left(H_{n}-z I_{n}\right)^{-1}\right]^{-1}\right\|_{n} \leqslant \frac{1}{1-M} .
$$

Так как, с другой стороны, в силу (14)

$$
\widetilde{E}_{n}\left[I_{n}+C_{n}\left(H_{n}-z I_{n}\right)^{-1}\right] E_{n}=\widetilde{E}_{n} E_{n}+\left(\widetilde{E}_{n} C_{n} E_{n}\right) \mathscr{R}_{n}^{(H)}(z) \rightarrow I+C \mathscr{R}^{(H)}(z),
$$


то можно применить лемму 5. Таким образом, получаем

$$
\widetilde{E}_{n}\left[I_{n}+C_{n}\left(H_{n}-z I_{n}\right)^{-1}\right]^{-1} E_{n} \rightarrow\left[I+C \mathscr{R}^{(H)}(z)\right]^{-1}
$$

Остается воспользоваться тождеством

$$
\mathscr{R}_{n}^{(G)}(z)=\mathscr{R}_{n}^{(H)}(z) \widetilde{E}_{n}\left[I_{n}+C_{n}\left(H_{n}-z I_{n}\right)^{-1}\right]^{-1} E_{n}
$$

Наконец, так как все резольвенты являются (операторнозначными) аналитическими функциями, равномерно ограниченньми на компактных подмножествах в указанной области, получаем равномерную сходимость. Теорема 1 доказана.

В предыдущем параграфе мы сформулировали некоторые следствия из теоремы 1. Следствия 1, 2 и 4 тривиальны; для доказательства следствия 3 достаточно установить следующий вспомогательный результат.

ЛЕмма 6. Если матрица $Н$ самосопряжена, то $H^{(k)}$ также самосопряжена для каждого $k \in \mathbb{N}$.

ДокАЗАтЕльство. Очевидно, достаточно доказать утверждение для $k=1$. Проверим симметричность максимального оператора $H^{(1)}$, т.е. проверим равенст$\mathrm{BO}$

$$
\left\langle H^{(1)} u^{(1)}, v^{(1)}\right\rangle=\left\langle u^{(1)}, H^{(1)} v^{(1)}\right\rangle \text { при } u^{(1)}, v^{(1)} \in D\left(H^{(1)}\right) .
$$

Для заданных $u^{(1)}=\left(u_{1}^{(1)}, u_{2}^{(1)}, \ldots\right), v^{(1)}=\left(v_{1}^{(1)}, v_{2}^{(1)}, \ldots\right) \in D\left(H^{(1)}\right)$ векторы $u=\left(0, u_{1}^{(1)}, u_{2}^{(1)}, \ldots\right)$ и $v=\left(0, v_{1}^{(1)}, v_{2}^{(1)}, \ldots\right)$ принадлежат $D(H)$ и имеет место равенство $\left\langle H^{(1)} u^{(1)}, v^{(1)}\right\rangle=\langle H u, v\rangle=\langle u, H v\rangle=\left\langle u^{(1)}, H^{(1)} v^{(1)}\right\rangle$, что и завершает доказательство.

ЗАмЕчАНИЕ 1. Из приведенного доказательства становится очевидньм, что аналогичный результат имеет место и для "разреженных" матриц, т.е. имеющих конечное число ненулевых элементов в каждом столбце и каждой строке.

\section{§3. Трехдиагональные матрицы и непрерывные дроби}

В этом параграфе мы изучим подробнее трехдиагональные матрицы из-за их связи с непрерывньми дробями. Точнее, рассмотрим бесконечную чебышевскую непрерывную дробь ( $J$-дробь $)$

$$
f(z)=\frac{1}{z-b_{0}-\frac{a_{1}^{2}}{z-b_{1}-\frac{a_{2}^{2}}{z-b_{2}-} \cdot}}
$$

при естественном условии

$$
a_{n} \neq 0, \quad n=1,2, \ldots
$$


и для каждого $n \in \mathbb{N}$ через

$$
f_{n}(z)=\frac{Q_{n}(z)}{P_{n}(z)}=\frac{1}{z-b_{0}-\frac{a_{1}^{2}}{z-b_{1}-\ddots \cdot-\frac{a_{n-1}^{2}}{z-b_{n-1}}}}
$$

обозначим ее $n$-ю подходящую дробь. При условии (17) $\operatorname{deg} P_{n}=n$, и можно считать, что старший коэффициент $P_{n}(z)$ равен единице. Легко проверяется хорошо известный факт, что многочлены $P_{n}$ и $Q_{n}$ удовлетворяют трехчленньм рекуррентньп соотношениям, а именно

$$
\begin{gathered}
P_{n+1}(z)=\left(z-b_{n}\right) P_{n}(z)-a_{n}^{2} P_{n-1}(z), \quad n \geqslant 1 \\
P_{0}(z)=1, \quad P_{1}(z)=z-b_{0}
\end{gathered}
$$

и

$$
\begin{gathered}
Q_{n+1}(z)=\left(z-b_{n}\right) Q_{n}(z)-a_{n}^{2} Q_{n-1}(z), \quad n \geqslant 1, \\
Q_{0}(z)=0, \quad Q_{1}(z)=1 .
\end{gathered}
$$

Это позволяет нам установить связь непрерывной дроби (16) с трехдиагональной матрицей

$$
G=\left(\begin{array}{cccc}
b_{0} & a_{1} & 0 & \ldots \\
a_{1} & b_{1} & a_{2} & \ldots \\
0 & a_{2} & b_{2} & \ldots \\
\vdots & \vdots & \vdots & \ddots
\end{array}\right)
$$

Точнее, используя (19), легко установить, что $P_{n}(z)=(-1)^{n} \operatorname{det}\left(G_{n}-z I_{n}\right), n \in \mathbb{N}$. Другими словами, множество нулей многочленов $P_{n}$, удовлетворяющих $(19)$, совпадает со спектром $\sigma\left(G_{n}\right)$.

В дальнейшем мы будем использовать аналогичные многочлены, связанные со “сдвинутой” матрицей $G^{(k)}$. Пусть

$$
P_{n}^{(k)}(z)=(-1)^{n} \operatorname{det}\left(G_{n}^{(k)}-z I_{n}\right), \quad n \in \mathbb{N}
$$

(в частности, $\left.P_{n}^{(0)}(z)=P_{n}(z)\right)$; тогда имеет место рекуррентное соотношение

$$
\begin{gathered}
P_{n+1}^{(k)}(z)=\left(z-b_{n+k}\right) P_{n}^{(k)}(z)-a_{n+k}^{2} P_{n-1}^{(k)}(z), \quad n \geqslant 1, \\
P_{0}^{(k)}(z)=1, \quad P_{1}^{(k)}(z)=z-b_{k} .
\end{gathered}
$$

Полезная формула, связываюшая многочлены, соответствующие различным сдвигам, получается разложением $\operatorname{det}\left(G_{n}^{(k)}-z I_{n}\right)$ по первой строке:

$$
P_{n}^{(k)}(z)=\left(z-b_{k}\right) P_{n-1}^{(k+1)}(z)-a_{k+1}^{2} P_{n-2}^{(k+2)}(z), \quad k \geqslant 0, \quad n \geqslant 1
$$


Некоторые формулы, приводимые ниже, будут вьглядеть проше при другой нормировке $P_{n}^{(k)}(z)$. Положим

$$
p_{n}^{(k)}(z)=\frac{P_{n}^{(k)}(z)}{a_{k} a_{k+1} \cdots a_{k+n}} \text { при } n \geqslant 0,
$$

считая $a_{0}=1$. Тогда $(22)$ можно представить в следуюшей форме

$$
\begin{gathered}
a_{n+k+1} p_{n+1}^{(k)}(z)=\left(z-b_{n+k}\right) p_{n}^{(k)}(z)-a_{n+k} p_{n-1}^{(k)}(z), \quad n \geqslant 1, \\
p_{0}^{(k)}(z)=\frac{1}{a_{k}}, \quad p_{1}^{(k)}(z)=\frac{z-b_{k}}{a_{k} a_{k+1}},
\end{gathered}
$$

a (23) принимает вид

$$
a_{k} p_{n}^{(k)}(z)=\left(z-b_{k}\right) p_{n-1}^{(k+1)}(z)-a_{k+1} p_{n-2}^{(k+2)}(z)
$$

(Другое доказательство этого тождества см., например, в [9].)

Из (22) и (20) очевидно следует, что $Q_{n} \equiv P_{n-1}^{(1)}$, поэтому (см. (18))

$$
f_{n}(z)=\frac{P_{n-1}^{(1)}(z)}{P_{n}(z)}=\frac{p_{n-1}^{(1)}(z)}{p_{n}(z)}
$$

где $p_{n}(z)=p_{n}^{(0)}(z)$.

Ключевым моментом в исследовании сходимости непрерывной дроби (16) в терминах операторов является следуюшее хорошо известное представление для подходящих дробей (см., например, [10; теорема 3.7, с. 131]):

$$
f_{n}(z)=-\left\langle\mathscr{R}_{n}^{(G)}(z) e_{0}, e_{0}\right\rangle, \quad n \in \mathbb{N}, \quad z \in \mathbb{C} \backslash \sigma\left(G_{n}\right)
$$

Таким образом, даже слабой сходимости резольвент $\mathscr{R}_{n}^{(G)}(z)$ достаточно для сходимости $f_{n}(z)$, а значит, теорема 1 , установленная в $\S 2$, имеет следующее непосредственное следствие.

Теорема 2. а) Если матрица $G$, определенная в (21), допускает разложение (2), то последовательность $f_{n}(z)$ равномерно сходится на компактах из $\{z \in \mathbb{C}: \operatorname{dist}(z, \operatorname{conv} \sigma(H))>\|C\|\} \kappa$ аналитической функиии

$$
f(z)=-\left\langle\mathscr{R}^{(G)}(z) e_{0}, e_{0}\right\rangle
$$

б) Для любой непрерывной дроби (16) с равномерно ограниченными комплексными коэффициентами

$$
f_{n}(z) \rightrightarrows f(z), \quad n \rightarrow \infty
$$

по крайней мере на компактах из $\{|z|>\|G\|\}$. 
ЗАмечание 2. Если в разложении (2)

$$
H=\left(\begin{array}{cccc}
\beta_{0} & \alpha_{1} & 0 & \cdots \\
\alpha_{1} & \beta_{1} & \alpha_{2} & \cdots \\
0 & \alpha_{2} & \beta_{2} & \cdots \\
\vdots & \vdots & \vdots & \ddots
\end{array}\right)
$$

то (29) имеет место на компактах $K$, удовлетворяющих условию

$$
\operatorname{dist}\left(K,\left[\gamma_{-}, \gamma_{+}\right]\right)>M
$$

где $\gamma_{-}=\liminf \left(\beta_{n}-\left|\alpha_{n}\right|-\left|\alpha_{n+1}\right|\right), \gamma_{+}=\limsup \left(\beta_{n}+\left|\alpha_{n}\right|+\left|\alpha_{n+1}\right|\right)$ и $M=$ $\sup \left(\left|b_{n}-\beta_{n}\right|+\left|a_{n}-\alpha_{n}\right|+\left|a_{n+1}-\alpha_{n+1}\right|\right)$. Эти границы непосредственно следуют из теоремы Гершгорина и из оценок операторной нормы матрицы (см., например, $[11 ;$ c. 71,72$])$.

ЗАмечание 3. Можно получить некоторое простое обобшение формулы (28). Решая соответствуюшую линейную систему, получаем

$$
p_{i-1}(z) \frac{p_{n-j}^{(j)}(z)}{p_{n}(z)}=-\left\langle\mathscr{R}_{n}^{(G)}(z) e_{j-1}, e_{i-1}\right\rangle, \quad n \in \mathbb{N}, \quad z \in \mathbb{C} \backslash \sigma\left(G_{n}\right) .
$$

Таким образом, теорема 1 позволяет установить несколько более сильный вариант теоремы 2 , который мы сформулируем в следующем виде:

СлеДСтвИЕ 5. В условиях а) теоремы 2

$$
p_{i-1}(z) \frac{p_{n-j}^{(j)}(z)}{p_{n}(z)} \rightrightarrows-\left\langle\mathscr{R}^{(G)}(z) e_{j-1}, e_{i-1}\right\rangle, \quad n \rightarrow \infty
$$

на компактах, лежащих в $\{z \in \mathbb{C}: \operatorname{dist}(z, \operatorname{conv} \sigma(H))>\|C\|\}$.

\section{§4. Компактные возмущения матриц Якоби}

В этом параграфе мы уточним полученные выше результаты о сходимости непрерывных дробей для частного случая, когда матрица $C=\left(c_{i j}\right)$ трех диагональна и компактна, что эквивалентно

$$
c_{i j}=0 \text { при }|i-j|>1, \quad \lim _{n \rightarrow \infty} c_{n+k, n}=0, \quad k \in \mathbb{Z} .
$$

Мы пользуемся введенными выше определениями и обозначениями.

Ключевой факт состоит в соотношении

$$
\lim _{k \rightarrow \infty}\left\|C^{(k)}\right\|=0
$$

вытекающем из (30). С его помощью мы можем "приблизиться" к действительной оси и установить более точные результаты даже вблизи вьпуклой оболочки 
$\sigma(H)$. Связь между резольвентными операторами для $G$ и $G^{(k)}$ доказывается с помошью следуюшей леммы, которую мы формулируем без доказательства в силу ее простоты.

Лемма 7. Пусть матрица

$$
M=\left(\begin{array}{cc}
A & B \\
B^{T} & C
\end{array}\right)
$$

является обратимой конечной матрицей, где $A, C$ - обратимье матриць, $A=A^{T}, C=C^{T}$. Тогда матрицы $A-B C^{-1} B^{T}$ и $C-B^{T} A^{-1} B$ обратимъ и

$$
M^{-1}=\left(\begin{array}{cc}
\left(A-B C^{-1} B^{T}\right)^{-1} & -A^{-1} B\left(C-B^{T} A^{-1} B\right)^{-1} \\
-C B^{T}\left(A-B C^{-1} B^{T}\right)^{-1} & \left(C-B^{T} A^{-1} B\right)^{-1}
\end{array}\right)
$$

Дополнительно нам необходимо более точное описание множества $\mathscr{P}(G)$ для случая компактного возмущения, полученное в [12; теорема 2].

Лемма 8. Имеем

$$
\mathscr{P}(G) \subset \sigma_{p}(G) \cup \bigcap_{k=0}^{\infty} \operatorname{conv} \sigma\left(H^{(k)}\right) .
$$

Теперь мы готовы к формулировке и доказательству основного результата настоящего параграфа.

Tеорема 3. Если имеет место (2) и матрица $C$ компактна и трехдиагональна, то $\mathscr{R}_{n}^{(G)}(z) \rightarrow \mathscr{R}^{(G)}(z)$ равномерно на компактных подмножествах в $\mathbb{C} \backslash\left[\sigma_{p}(G) \cup \bigcap_{k=0}^{\infty} \operatorname{conv} \sigma\left(H^{(k)}\right)\right]$.

ДоКАЗАТЕЛЬСтво. Установим в первую очередь ограниченность последовательности $\left\{\left\|\mathscr{R}_{n}^{(G)}(z)\right\|, n \geqslant n_{0}(z)\right\}$ для каждого $z \in \mathbb{C} \backslash\left[\sigma_{p}(G) \cup \bigcap_{k=0}^{\infty} \operatorname{conv} \sigma\left(H^{(k)}\right)\right]$. Для упрощения обозначений в дальнейшем будем отождествлять конечномерные операторы с их матричным представлением.

Для данного $z \notin \sigma_{p}(G) \cup \bigcap_{k=0}^{\infty} \operatorname{conv} \sigma\left(H^{(k)}\right)$ можно найти $k_{1} \in \mathbb{N}$, удовлетворяюшее неравенству

$$
\operatorname{dist}\left(z, \mathscr{P}\left(H^{(k)}\right) \cup \sigma\left(H^{(k)}\right)\right) \geqslant \operatorname{dist}\left(z, \operatorname{conv} \sigma\left(H^{\left(k_{1}\right)}\right)\right)
$$

для любого $k \geqslant k_{1}$ (заметим, что $\left.\operatorname{conv} \sigma\left(H^{(k)}\right) \subset \operatorname{conv} \sigma\left(H^{\left(k_{1}\right)}\right), k \geqslant k_{1}\right)$. Так как

$$
\operatorname{dist}\left(z, \operatorname{conv} \sigma\left(H^{\left(k_{1}\right)}\right)\right)>0
$$

то таким же образом находим $\widetilde{k}_{1} \in \mathbb{N}$, удовлетворяющее неравенству

$$
\operatorname{dist}\left(z, \operatorname{conv} \sigma\left(H^{\left(k_{1}\right)}\right)\right)>\left\|C^{(k)}\right\|
$$


для любого $k \geqslant \widetilde{k}_{1}$. Следовательно, существует $k_{0}=\max \left\{k_{1}, \widetilde{k}_{1}\right\}$ такое, что

$$
\operatorname{dist}\left(z, \mathscr{P}\left(H^{(k)}\right) \cup \operatorname{conv} \sigma\left(H^{(k)}\right)\right)>\left\|C^{(k)}\right\|
$$

для любого $k \geqslant k_{0}$. Но тогда в силу следствия 3 существует такое $n_{0}=n_{0}(z, k) \in \mathbb{N}$, что как $G^{(k)}-z I$, так и $G_{n}^{(k)}-z I_{n}$ являются обратимьми для каждого $n \geqslant n_{0}$. Кроме того, для таких $k$ последовательность $\left\{\left(G_{n}^{(k)}-z I_{n}\right)^{-1}\right\}_{n \geqslant n_{0}}$ ограничена по норме.

С другой стороны, лемма 8 утверждает, что существует $n_{1}=n_{1}(z)$ такое, что $G_{n}-z I_{n}$ обратим при $n \geqslant n_{1}$. Фиксируем $k \geqslant \max \left\{k_{0}, n_{1}\right\}$ и пусть $n \geqslant k+n_{0}$; тогда матрицы $G_{k}-z I_{k}, G_{n-k}^{(k)}-z I_{n-k}, G_{n}-z I_{n}$ также обратимы. Применяя лемму 7 к матрице $M=G_{n}-z I_{n}$, получаем

$$
\left(G_{n}-z I_{n}\right)^{-1}=\left(\begin{array}{cc}
D_{n, k}^{-1} & -\left(G_{k}-z I_{k}\right)^{-1} B_{k} F_{n, k}^{-1} \\
-\left(G_{n-k}^{(k)}-z I_{n-k}\right)^{-1} B_{k}^{T} D_{n, k}^{-1} & F_{n, k}^{-1}
\end{array}\right),
$$

где $D_{n, k}=\left(G_{k}-z I_{k}\right)-B_{k}\left(G_{n-k}^{(k)}-z I_{n-k}\right)^{-1} B_{k}^{T}$ и $F_{n, k}=\left(G_{n-k}^{(k)}-z I_{n-k}\right)-$ $B_{k}^{T}\left(G_{k}-z I_{k}\right)^{-1} B_{k}-$ обратимые матрицы порядка $k \times k$ и $(n-k) \times(n-k)$ соответственно, а

$$
B_{k}=a_{k}\left(\begin{array}{ccc}
0 & \cdots & 0 \\
\vdots & & \vdots \\
0 & \cdots & 0 \\
1 & \cdots & 0
\end{array}\right)
$$

является конечной матрицей порядка $k \times(n-k)$. Так как операторная норма $\left\|B_{k}\right\|=\left|a_{k}\right|$ для всех $n$, достаточно показать равномерную ограниченность норм блоков $D_{n, k}^{-1}$ и $F_{n, k}^{-1}$. Докажем это в два шага.

а) Для оценки $D_{n, k}^{-1}$ заметим, что для

$$
\left(G_{n-k}^{(k)}-z I_{n-k}\right)^{-1}=\left(\begin{array}{ccc}
\alpha_{1,1} & \cdots & \alpha_{1, n-k} \\
\vdots & & \vdots \\
\alpha_{n-k, 1} & \cdots & \alpha_{n-k, n-k}
\end{array}\right)
$$

непосредственно проверяется равенство

$$
B_{k}\left(G_{n-k}^{(k)}-z I_{n-k}\right)^{-1} B_{k}^{T}=a_{k}^{2} \alpha_{1,1}\left(\begin{array}{cccc}
0 & & & \\
& \ddots & & \\
& & 0 & \\
& & & 1
\end{array}\right)
$$

где $\alpha_{1,1}=\left\langle\mathscr{R}_{n-k}^{\left(G^{(k)}\right)}(z) e_{0}, e_{0}\right\rangle=-f_{n-k}^{(k)}(z)$ (см. (36) и (28)). Следовательно, $D_{n, k}$ является трех диагональной матрицей, все элементы которой совпадают с элементами $G_{k}-z I_{k}$, кроме последнего элемента на главной диагонали. Другими словами,

$$
D_{n, k}=\left(\begin{array}{ccccc}
b_{0}-z & a_{1} & \ldots & 0 & 0 \\
a_{1} & b_{1}-z & \ldots & 0 & 0 \\
\vdots & \vdots & \ddots & \vdots & \vdots \\
0 & 0 & \ldots & b_{k-2}-z & a_{k-1} \\
0 & 0 & \ldots & a_{k-1} & b_{k-1}-z+a_{k}^{2} f_{n-k}^{(k)}(z)
\end{array}\right)
$$


Значит, операторы $D_{n, k}: \mathbb{C}^{k} \rightarrow \mathbb{C}^{k}$ сходятся к $D_{k}: \mathbb{C}^{k} \rightarrow \mathbb{C}^{k}$, определяемому формулой

$$
D_{k}=\left(\begin{array}{ccccc}
b_{0}-z & a_{1} & \ldots & 0 & 0 \\
a_{1} & b_{1}-z & \ldots & 0 & 0 \\
\vdots & \vdots & \ddots & \vdots & \vdots \\
0 & 0 & \ldots & b_{k-2}-z & a_{k-1} \\
0 & 0 & \ldots & a_{k-1} & b_{k-1}-z+a_{k}^{2} f^{(k)}(z)
\end{array}\right)
$$

Так как мы имеем дело с ограниченными операторами в $\mathbb{C}^{k}$ ( $k$ фиксировано), мы получим равномерную ограниченность, просто установив обратимость $D_{k}$, т.е. невырожденность его матрицы. Но, принимая во внимание (19), так же как в [12; теорема 3] приходим к соотношению

$$
\operatorname{det}\left(D_{k}\right)=(-1)^{k}\left[P_{k}(z)-a_{k}^{2} P_{k-1} f^{(k)}(z)\right] \neq 0
$$

б) Для оценки $F_{n, k}^{-1}$ запишем

$$
\left(G_{k}-z I_{k}\right)^{-1}=\left(\begin{array}{ccc}
\gamma_{1,1} & \cdots & \gamma_{1, k} \\
\vdots & & \vdots \\
\gamma_{k, 1} & \cdots & \gamma_{k, k}
\end{array}\right)
$$

тогда непосредственно проверяется, что

$$
B_{k}^{T}\left(G_{k}-z I_{k}\right)^{-1} B_{k}=a_{k}^{2} \gamma_{k, k}\left(\begin{array}{cccc}
1 & & & \\
& 0 & & \\
& & \ddots & \\
& & & 0
\end{array}\right)
$$

является диагональной матрицей порядка $n-k$.

Таким образом, как и раньше, $F_{n, k}$ является трехдиагональной матрицей, совпадающей с $G_{n-k}^{(k)}-z I_{n-k}$ всюду, за исключением первого элемента, но теперь размер каждого блока меняется вместе с $n$. В этом случае необходимы более тонкие вычисления.

Так как $\left(G_{n-k}^{(k)}-z I_{n-k}\right)-F_{n, k}=B_{k}^{T}\left(G_{k}-z I_{k}\right)^{-1} B_{k}$, получаем

$$
F_{n, k}^{-1}-\left(G_{n-k}^{(k)}-z I_{n-k}\right)^{-1}=\left(G_{n-k}^{(k)}-z I_{n-k}\right)^{-1} B_{k}^{T}\left(G_{k}-z I_{k}\right)^{-1} B_{k} F_{n, k}^{-1}
$$

Значит,

$$
\left\|F_{n, k}^{-1}\right\| \leqslant\left\|\left(G_{n-k}^{(k)}-z I_{n-k}\right)^{-1}\right\|\left[1+\left\|B_{k}^{T}\left(G_{k}-z I_{k}\right)^{-1} B_{k} F_{n, k}^{-1}\right\|\right]
$$


В силу того что $\left\{\left\|\left(G_{n-}^{(k)}-z I_{n-k}\right)^{-1}\right\|, n \geqslant k+n_{0}\right\}$ является ограниченной последовательностью, мы приходим к заключению, что достаточно установить ограниченность последовательности $\left\{\left\|B_{k}^{T}\left(G_{k}-z I_{k}\right)^{-1} B_{k} F_{n, k}^{-1}\right\|, n \geqslant k+n_{0}\right\}$.

С другой стороны, записывая

$$
\left(G_{n-k}^{(k)}-z I_{n-k}\right)^{-1}=\frac{1}{\operatorname{det}\left(G_{n-k}^{(k)}-z I_{n-k}\right)}\left(\begin{array}{cccc}
g_{1,1} & g_{1,2} & \cdots & g_{1, n-k} \\
g_{2,1} & g_{2,2} & \cdots & g_{2, n-k} \\
\vdots & \vdots & & \vdots \\
g_{n-k, 1} & g_{n-k, 2} & \cdots & g_{n-k, n-k}
\end{array}\right)
$$

$\left(g_{i, j}=\alpha_{i, j} \operatorname{det}\left(G_{n-k}^{(k)}-z I_{n-k}\right), \quad i, j=1, \ldots, n-k\right)$ и принимая во внимание структуру $F_{n, k}$, получаем

$$
\begin{gathered}
F_{n, k}^{-1}=\frac{1}{\operatorname{det}\left(F_{n, k}\right)}\left(\begin{array}{cccc}
g_{1,1} & g_{1,2} & \cdots & g_{1, n-k} \\
g_{2,1} & f_{2,2} & \cdots & f_{2, n-k} \\
\vdots & \vdots & & \vdots \\
g_{n-k, 1} & f_{n-k, 2} & \cdots & f_{n-k, n-k}
\end{array}\right), \\
f_{i, j} \in \mathbb{C}, \quad i, j=2, \ldots, n-k .
\end{gathered}
$$

Далее, из (42) следует, что

$$
\begin{aligned}
B_{k}^{T}\left(G_{k}-z I_{k}\right)^{-1} B_{k} F_{n, k}^{-1} & \\
= & \frac{a_{k}^{2} \gamma_{k, k}}{\operatorname{det}\left(F_{n, k}\right)}\left(\begin{array}{cccc}
g_{1,1} & g_{1,2} & \cdots & g_{1, n-k} \\
0 & 0 & \cdots & 0 \\
\vdots & \vdots & & \vdots \\
0 & 0 & \cdots & 0
\end{array}\right) \\
= & \frac{\operatorname{det}\left(G_{n-k}^{(k)}-z I_{n-k}\right)}{\operatorname{det}\left(F_{n, k}\right)} B_{k}^{T}\left(G_{k}-z I_{k}\right)^{-1} B_{k}\left(G_{n-k}^{(k)}-z I_{n-k}\right)^{-1}
\end{aligned}
$$

Следовательно, для доказательства равномерной ограниченности блоков $F_{n, k}^{-1}$ достаточно установить это для последовательности

$$
\left\{\frac{\operatorname{det}\left(G_{n-k}^{(k)}-z I_{n-k}\right)}{\operatorname{det}\left(F_{n, k}\right)}\right\}_{n \geqslant k+n_{0}} .
$$


Разлагая $\operatorname{det}\left(F_{n, k}\right)$ по первой строке и принимая во внимание трехдиагональную структуру матрицы, получим

$$
\begin{aligned}
\operatorname{det}\left(F_{n, k}\right) & =\operatorname{det}\left(\begin{array}{ccccc}
b_{k}-z-a_{k}^{2} \gamma_{k, k} & a_{k+1} & \ldots & 0 & 0 \\
a_{k+1} & b_{k+1}-z & \ldots & 0 & 0 \\
\vdots & \vdots & \ddots & \vdots & \vdots \\
0 & 0 & \ldots & b_{n-2}-z & a_{n-1} \\
0 & 0 & \ldots & a_{n-1} & b_{n-1}-z
\end{array}\right) \\
& =\operatorname{det}\left(G_{n-k}^{(k)}-z I_{n-k}\right)-a_{k}^{2} \gamma_{k, k} \operatorname{det}\left(G_{n-k-1}^{(k+1)}-z I_{n-k-1}\right) \\
& =(-1)^{n-k} P_{n-k}^{(k)}(z)-a_{k}^{2} \gamma_{k, k}(-1)^{n-k-1} P_{n-k-1}^{(k+1)}(z),
\end{aligned}
$$

где $\gamma_{k, k}=-P_{k-1}(z) / P_{k}(z)$. Значит, последовательность

$$
\begin{aligned}
\frac{\operatorname{det}\left(G_{n-k}^{(k)}-z I_{n-k}\right)}{\operatorname{det}\left(F_{n, k}\right)} & =\frac{P_{n-k}^{(k)}(z)}{P_{n-k}^{(k)}(z)-a_{k}^{2} \frac{P_{k-1}(z)}{P_{k}(z)} P_{n-k-1}^{(k+1)}(z)} \\
& =\frac{P_{k}(z)}{P_{k}(z)-a_{k}^{2} P_{k-1}(z) f_{n-k}^{(k)}(z)}
\end{aligned}
$$

стремится при $n \rightarrow \infty \mathrm{K}$

$$
\frac{P_{k}(z)}{P_{k}(z)-a_{k}^{2} P_{k-1}(z) f^{(k)}(z)} \neq \infty
$$

(cм. (40)).

Таким образом, мы установили ограниченность норм конечномерных резольвент для каждого фиксированного $z \in \mathbb{C} \backslash\left[\sigma_{p}(G) \cup \bigcap_{k=0}^{\infty} \operatorname{conv} \sigma\left(H^{(k)}\right)\right]$. Для завершения доказательства остается установить сходимость на плотном подмножестве $\ell^{2}$. Мы следуем схеме, аналогичной уже использованной нами при доказательстве леммы 4.

Пусть $v_{j}=(G-z I) e_{j}, j=0,1, \ldots ;$ необходимо установить плотность $\operatorname{span}\left\{v_{j}\right.$, $j=0,1, \ldots\}$ в $\ell^{2}$. Несмотря на то что теперь, вообше говоря, $G$ несамосопряжен, имеет место соотношение $g_{i j}=g_{j i}$ (см. (1)), откуда следует, что $x=0$ - это единственный вектор из $D(G)$, ортогональный к $\operatorname{span}\left\{v_{j}, j=0,1, \ldots\right\}$.

Поточечная сходимость на векторах $v_{i}$ проверяется непосредственно, а равномерная сходимость получается как в доказательстве леммы 4.

ЗАмЕчАниЕ 4. Предыдущий результат может иметь самостоятельный интерес, так как дает нам матричное представление для $\mathscr{R}^{(G)}(z)$. Достаточно лишш воспользоваться поблочной сходимостью матрицы в (34).

\section{СлЕДСТВИЕ 6. Имеем}

$$
f_{n}(z) \rightrightarrows f(z)=-\left\langle\mathscr{R}^{(G)}(z) e_{0}, e_{0}\right\rangle \quad n p u \quad n \rightarrow \infty
$$

на любом компактном подмножестве в $\mathbb{C} \backslash\left[\sigma_{p}(G) \cup \bigcap_{k=0}^{\infty} \operatorname{conv} \sigma\left(H^{(k)}\right)\right]$. 
ДоКАЗАТЕЛЬСТВо. Достаточно Учесть, что

$$
\left|f_{n}(z)-f(z)\right|=\left|\left\langle\mathscr{R}_{n}^{(G)}(z) e_{0}-\mathscr{R}^{(G)}(z) e_{0}, e_{0}\right\rangle\right| \leqslant\left\|\mathscr{R}_{n}^{(G)}(z) e_{0}-\mathscr{R}^{(G)}(z) e_{0}\right\| .
$$

В заключение мы установим связь между полюсами $f(z)$, полюсами ее подходящих дробей и собственными значениями $G$. Воспользуемся следуюшим результатом (см. [12; лемма 4]):

Лемма 9. В условиях теоремы 3

$$
\left(\sigma_{p}\left(G^{(m)}\right) \cap \sigma_{p}\left(G^{(m+1)}\right)\right) \backslash \bigcap_{k=0}^{\infty} \operatorname{conv} \sigma\left(H^{(k)}\right)=\varnothing
$$

для любъх $m \geqslant 0$.

Всюду в дальнейшем для произвольного множества $U \subset \mathbb{C}$ через $\varkappa\left(P_{n}, U\right)$ обозначим число нулей (с учетом кратностей) $P_{n}$ в $U$.

Tеорема 4. Функиия $f$ мероморфна в $\mathbb{C} \backslash \bigcap_{k=0}^{\infty} \operatorname{conv} \sigma\left(H^{(k)}\right)$, при этом каждая точка $\sigma_{p}(G) \backslash \bigcap_{k=0}^{\infty} \operatorname{conv} \sigma\left(H^{(k)}\right)$ является полюсом $f$. Кроме того,

$$
\varkappa(\zeta)=m(\zeta, G)
$$

для всех $\zeta \in \sigma_{p}(G)$, где $\varkappa(\zeta)$ обозначает порядок $\zeta$ как полюса $f$, а $m(\zeta, G)$ обозначает алгебрачческую кратность $\zeta$ как собственного значения $G$.

ДокАЗАтЕльство. Фиксируем $\zeta \in \sigma_{p}(G) \backslash \bigcap_{k=0}^{\infty} \operatorname{conv} \sigma\left(H^{(k)}\right)$. Как известно, $\zeta$ должна быть изолированной точкой $\sigma_{p}(G)$, а следовательно, сушествует $\delta>0$ такое, что

$$
U(\zeta, \delta) \stackrel{\text { def }}{=}\{z: 0<|z-\zeta| \leqslant \delta\} \subset \rho(G) \backslash \bigcap_{k=0}^{\infty} \operatorname{conv} \sigma\left(H^{(k)}\right) .
$$

Кроме того, из леммы 9 известно, что $\zeta \notin \sigma_{p}\left(G^{(1)}\right)$, поэтому можно считать, что $\delta$ достаточно мало и удовлетворяет условию $\overline{U(\zeta, \delta)} \cap \sigma\left(G^{(1)}\right)=\varnothing$. Таким образом, $P_{n-1}^{(1)}(z) \neq 0$ при $z \in U(\zeta, \delta)$ и $n \geqslant n_{1}(U(\zeta, \delta))$ (в противном случае должна существовать предельная точка нулей $\left\{P_{n}^{(1)}\right\}_{n \in \mathbb{N}}$ в $\overline{U(\zeta, \delta)}$, что противоречит лемме 8).

Если положить $\Gamma \stackrel{\text { def }}{=}\{z:|z-\zeta|=\delta\}$, то из равномерной сходимости $f_{n}$ на $\Gamma$ (следствие 6) следует

$$
\lim _{n \rightarrow \infty} \frac{1}{2 \pi i} \int_{\Gamma} \frac{f_{n}^{\prime}(z)}{f_{n}(z)} d z=\frac{1}{2 \pi i} \int_{\Gamma} \frac{f^{\prime}(z)}{f(z)} d z .
$$

Используя принцип аргумента и принимая во внимание, что интегралы в левой части целочисленны, приходим к заключению, что

$$
\varkappa\left(P_{n}, U(\zeta, \delta)\right)=\varkappa(\zeta) \text { при } n \geqslant n_{2}(U(\zeta, \delta)) .
$$


$\mathrm{C}$ другой стороны, с помощью леммы 8 находим такое $n_{2}=n_{2}(\Gamma)$, что $\Gamma \subset \rho\left(G_{n}\right)$ при $n \geqslant n_{2}$. Таким образом, можно определить линейные операторы на $\ell^{2}$

$$
P(\zeta, \Gamma)=-\frac{1}{2 \pi i} \int_{\Gamma} \mathscr{R}^{(G)}(z) d z, \quad P_{n}(\zeta, \Gamma)=-\frac{1}{2 \pi i} \int_{\Gamma} \mathscr{R}_{n}^{(G)}(z) d z, \quad n \geqslant n_{2}
$$

Предварительно была установлена равномерная сходимость $\mathscr{R}_{n}^{(G)}(z)$ к $\mathscr{R}^{(G)}(z)$ на компактах, откуда

$$
P_{n}(\zeta, \Gamma) \rightarrow P(\zeta, \Gamma)
$$

Известно (см. [6; теорема 6.17 и следствия]), что при наших условиях

$$
\operatorname{dim} P(\zeta, \Gamma)\left(\ell^{2}\right)=m(\zeta, G) \quad \text { и } \operatorname{dim} P_{n}(\zeta, \Gamma)\left(\ell^{2}\right)=\varkappa\left(P_{n}, U(\zeta, \delta)\right)=\varkappa(\zeta)
$$

Более того, $\zeta$ является возможным полюсом $\mathscr{R}^{(G)}(z)$, порядок которого не превышает $m(\zeta, G)$. Следовательно,

$$
\mathscr{R}^{(G)}(z)=\sum_{\nu=-m(\zeta, G)}^{\infty}(z-\zeta)^{\nu} B_{\nu}
$$

$B_{\nu}$ - ограниченные операторы, причем некоторые $B_{\nu}$ могут быть равны нулю для $\nu \geqslant-m(\zeta, G)$ (см. также [13; теорема 1.3, с. 26, и теорема 2.2, с. 326]). Отсюда и из (47) в силу следствия 6 получаем

$$
f(z)=-\sum_{\nu=-m(\zeta, G)}^{\infty}(z-\zeta)^{\nu}\left\langle B_{\nu} e_{0}, e_{0}\right\rangle
$$

Но порядок $\zeta$ как полюса $f$ равен $\varkappa(\zeta)$, откуда $\varkappa(\zeta) \leqslant m(\zeta, G)$. Осталось лишь воспользоваться следующей леммой, которую мы приводим для полноты изложения:

Лемма 10 [6; с. 438]. Пусть $\left\{P_{n}\right\}, n=1,2, \ldots$, является последовательностью проекиий в банаховом пространстве $\mathbb{X}$, удовлетворяющей условию: $P_{n} \rightarrow P$, где $P$ - линейный ограниченный оператор на $\mathbb{X}$. Тогда $P$ также является проекиией. Допустим в дополнение, что $\operatorname{dim} P_{n} \leqslant \operatorname{dim} P<\infty$ для всех п. Тогда

$$
\operatorname{dim} P_{n}=\operatorname{dim} P
$$

для всех достаточно больших п.

Принимая во внимание (50) и (51), получаем теперь (48). Теорема доказана. 


\section{Список литературы}

1. Ахиезер Н. И. Классическая проблема моментов. М.: Физматгиз, 1961.

2. Никишин E. М., Сорокин B. Н. Рациональнье аппроксимации и ортогональность. М.: Наука, 1988.

3. Dombrowski J. Orthogonal polynomials and functional analysis // Orthogonal polynomials: theory and practice. Proc. NATO ASI, Colombus/OH (USA) 1989, NATO ASI Ser., Ser. C. V. 294, 1990. P. 147-161.

4. Аллахвердиев Б. П., Гусейнов Г. Ш. К спектральной теории диссипативных разностных операторов второго порядка // Матем. сб. 1989. Т. 180. № 1. С. 101-118.

5. Chihara T. S. An introduction to orthogonal polynomials. New York: Gordon and Breach, 1978.

6. Kato T. Perturbation theory of linear operators. Berlin: Springer-Verlag, 1976.

7. Kantorovich L. V., Akilov G. P. Functional analysis in normed spaces. Oxford: Pergamon Press, 1964.

8. Magnus A. P. Toeplitz matrix techniques and convergence of complex weight Padéapproximants // J. Comput. Appl. Math. 1987. V. 19. P. 23-38.

9. Van Assche $W$. Orthogonal polynomials, associated polynomials and functions of the second kind // J. Comput. Appl. Math. 1991. V. 37. P. 237-249.

10. Brezinski $C$. Padé-type approximation and general orthogonal polynomials. Basel: Birkhäuser, 1980.

11. Wilkinson J. H. The algebraic eigenvalue problem. Oxford: Oxford University Press, 1965.

12. Barrios D., López G., Martínez A., Torrano E. On the domain of convergence and poles of $J$-fractions // J. Approx. Theory. 1998. V. 93. № 2. P. 177-200.

13. Gohberg I., Goldberg S., Kaashoek M. A. Classes of linear operators. V. 5. Basel: Birkhäuser, 1990.

Мадридский Политехнический Университет, Испания;

Университет Carlos III, Мадрид, Испания;

Университет Альмерии, Испания;

Мадридский Политехнический Университет, Испания
Поступила в редакцию 25.12 .1995 и 15.06 .1998 